\title{
A RELATIONSHIP BETWEEN DEPOSITION VELOCITY AND TRAJECTORY REFLECTION PROBABILITY FOR USE IN STOCHASTIC LAGRANGIAN DISPERSION MODELS
}

\author{
J.D. WILSON ${ }^{1}$, F.J. FERRANDINO ${ }^{2}$ and G.W. THURTELL ${ }^{3}$ \\ 'Geography Department, University of Alberta, Edmonton, Alb. (Canada) \\ ${ }^{2}$ Department of Plant Pathology and Ecology, Connecticut Agricultural Experiment Station, \\ New Haven, CT (U.S.A.) \\ ${ }^{3}$ Department of Land Resource Science, University of Guelph, Guelph, Ont. (Canada)
}

(Received October 17, 1988; revision accepted January 31, 1989)

\begin{abstract}
Wilson, J.D., Ferrandino, F.J. and Thurtell, G.W., 1989. A relationship between deposition velocity and trajectory reflection probability for use in stochastic Langrangian dispersion models. Agric. For. Meteorol., 47: 139-154.

This paper is concerned with the efficient treatment of a partially absorbing surface within stochastic Lagrangian dispersion models. Near and within vegetation, the Lagrangian time scale is small, necessitating the use of a small time step. Much computational effort is expended in calculating path segments into and out of the canopy, although little streamwise displacement occurs (because of the low velocities in the canopy). For some purposes, it may be satisfactory to consider the gaseous elements to be reflected or absorbed some distance away from the surface. Our aim is to relate the trajectory reflection probability to the deposition velocity which is widely used to parameterise the depositional process. A relationship between the deposition velocity and the trajectory reflection probability is derived, and simulations using this reflection rule are shown to give the correct depositional flux and surface concentration for the cases tested, a finite elevated area source in the neutral surface layer and an elevated area source of infinite extent in homogeneous turbulence.
\end{abstract}

\section{INTRODUCTION}

The deposition of a gaseous substance from the atmosphere to an underlying reactive surface (see Fig. 1) is usually parameterised superficially by introducing the notion of a deposition velocity $w_{d}$ (or its reciprocal resistance, $r_{d}$ ). The flux density of the substance "to the surface", $F_{r}$, is defined as the magnitude of the mean flux density $\langle w c\rangle$ (where $w$ is the Eulerian velocity normal to the surface and $c$ the concentration; the angle bracket implies the average value) at a reference plane, $z_{r}$, lying above the surface cover. Provided lateral gradients in the horizontal fluxes are small (e.g., far downstream from the source), 


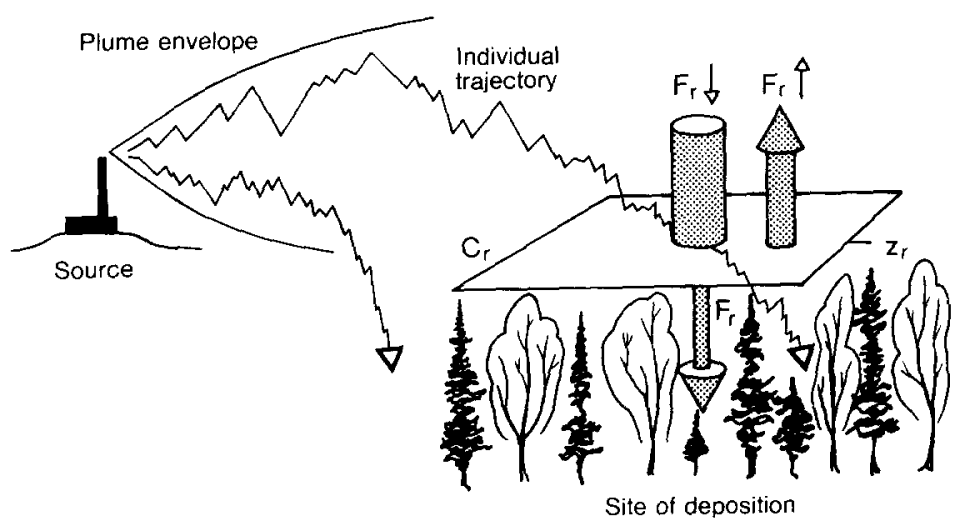

Fig. 1. Definitional sketch showing a reference plane at height $z=z_{r}$ above vegetation. A plume of gaseous pollutant from a distant source is being depleted by deposition to the soil and vegetation. An individual molecule passing across the reference plane may re-emerge or be captured. The deposition velocity, $w_{d}$, is defined in terms of the mean concentration, $C_{r}$, and the magnitude, $F_{r}$, of the mean flux density at the reference height, $F_{r}=w_{d} C_{r}$. Also shown are the conditional fluxes $F_{r} \downarrow$ and $F_{r} \uparrow$.

$F_{r}$ will closely approximate the absorption below $z_{r}$. The depositional flux will in general depend on many (not necessarily independent) variables describing the state of the atmosphere, the state of the vegetation and the state of the underlying soil (or artificial surface where involved). The deposition velocity and deposition resistance are defined by

$F_{r}=C_{r} w_{d}=C_{r} / r_{d}$

where $C_{r}=\left\langle c\left(z_{r}\right)\right\rangle$ is the mean concentration of the substance at the reference level $z_{r}$. All the details of the uptake process are lumped into the definition of $w_{d}$, whose magnitude therefore depends on the details of the process and also the choice of reference level $z_{r}$ (Ferrandino and Aylor, 1985). The concept of a deposition velocity is useful only when the diffusion pathway has an endpoint at which the concentration of the substance is zero (Chamberlain, 1966, 1967).

This simple model of surface uptake is widely used in calculations of the long-range transport of atmospheric pollutants (Smith, 1983; Fisher, 1983), and depends on prior determination of the deposition velocity appropriate to the underlying surface and the prevailing micrometeorological conditions.

Whether the range of interest be long or short, Lagrangian stochastic ("particle trajectory" /"random flight") models of dispersion (given the flow statistics) are in principle most readily able to encapsulate the complexity of the meteorological situation, the source distribution and the underlying surface. In practice, application of this approach (for other than short-range problems) 
will often be prohibited by the large computation time needed to calculate enough trajectories, especially if it is necessary to follow trajectories in and out of a plant or forest canopy where the very small Lagrangian time scale necessitates a correspondingly small time step. Furthermore, the accuracy of such simulations will in general be compromised by a lack of detailed knowledge of the velocity statistics (and perhaps by deficiency in the formulation of the random paths). Nevertheless, these models and the underlying theory are undergoing rapid development (e.g., Van Dop et al., 1985; Sawford, 1986; Sawford and Guest, 1987; Thomson, 1987).

The purpose of this paper is to formulate and discuss methods of incorporating surface uptake (either in bulk to a "surface" underlying a reference plane, or more microscopically to individual leaves or canopy layers) into Lagrangian stochastic models. The underlying experimental input continues to be the effective deposition velocity. The most efficient method involves partial reflection of fluid element trajectories at the reference level. This is straightforward to implement in a stochastic model, but some difficult theoretical questions must be answered. Clearly, the value of the reflection probability, $R$, must depend at least upon the specified deposition velocity. However, there are many possible implementations of a partial reflection strategy; for example, the reflection probability may or may not be allowed to depend on the magnitude of the downward velocity, $w_{\mathrm{L}}$, with which the particle arrives at the reflection site. A satisfactory theory should give an unambiguous definition of the reflection probability, i.e., a definition which specifies how reflection is to be implemented. The theory should furthermore determine whether and how the reflection process and probability depend upon the nature of the system of interest (e.g., neutral surface layer or homogeneous turbulence) and the discrete stochastic process chosen as a model for particle motion in that system.

The reflection probability for heavy particle trajectories will not be derived here. Reflection strategies for that case are given by Boughton et al. (1987) and by Aylor and Ferrandino (1989).

A reflection procedure and probability which we believe to have general, though perhaps not universal applicability is derived and discussed. An extended-sink treatment derived earlier by Boughton et al. is given with a simple alternative extended-sink treatment, both of which probably have restricted generality. Finally, results of numerical simulations which confirm the usefulness of the new reflection rule are given.

\section{"DIFFUSION LAYER" TREATMENT OF AN ABSORBING LOWER BOUNDARY FOR LAGRANGIAN MODELS}

Given the transfer resistance, $r_{d}$, between the physical location $z_{r}$ on the transfer pathway and the zero-concentration "endpoint" (wherever in space that may be located; the concept of a physical location for the endpoint may not be meaningful), we may place below $z=z_{r}$ a layer of depth 
$d=\sigma_{u}{ }^{2} T_{\mathrm{L}} r_{d}$

with a perfectly absorbing surface at $z=z_{r}-d$. This layer is characterised by velocity statistics $\sigma_{w}, T_{\mathrm{L}}$ and an effective diffusivity $K=d / r_{d}=\sigma_{w}{ }^{2} T_{\mathrm{L}}$. Here, $\sigma_{w}$ and $T_{\mathrm{L}}$ are the standard deviation and the decorrelation time scale for the Lagrangian vertical velocity. A particle passing below $z=z_{r}$ continues to move along the vertical with appropriate statistics and eventually is either absorbed at $z_{r}-d$ or re-emerges with $z>z_{r}$.

This is a very inefficient treatment of the absorption process, but will yield a relationship between flux and concentration at $z=z_{r}$ in correspondence with eq. 1 .

\section{DERIVATION OF THE TRAJECTORY REFLECTION PROBABILITY}

A reflection rule will now be derived without specification of the stochastic model into which the rule is to be imbedded. The rule and ways to implement it will then be discussed and it will be seen that the same rule arises from a more rigorous derivation in which the model is specified to be a Markovian joint velocity-position process, with what will be called "smooth wall" reflection. The joint velocity-position process is an appropriate model for the calculation of trajectories in both homogeneous and inhomogeneous turbulence.

\section{Derivation using mean fluxes conditioned on Eulerian velocity}

The mean vertical flux density along the normal to the partially absorbing surface $\langle w c\rangle$ may be decomposed as

$\langle w c\rangle=F \uparrow-F \downarrow$

where $f_{w}$ is the probability density function for the Eulerian vertical velocity. $F \uparrow$ and $F \downarrow$ are conditional mean fluxes defined by

$F \uparrow=\langle(w c \mid w>0)\rangle=\int_{0}^{\infty}\left\langle w c \mid w=w_{1}\right\rangle f_{w}\left(w_{1}\right) d w_{1}$

$F \downarrow=-\langle(w c \mid w<0)\rangle=-\int_{-\infty}^{0}\left\langle w c \mid w=w_{1}\right\rangle f_{w}\left(w_{1}\right) d w_{1}$

Now the depositional flux is $F_{r}=-\langle w c\rangle_{z=z r}=C_{r} w_{d}$, so that $-F_{r}=F_{r} \uparrow-F_{r} \downarrow$. Note that the definitions have been arbitrarily chosen so as to yield $F_{r}, F_{r} \uparrow$, $F_{r} \downarrow$ all positive.

The reflection probability, $R$, we wish to determine will be defined by the equation

$F_{r}=(1-R) F_{r} \downarrow$ 
so that

$1-R=C_{r} w_{d} / F_{r} \downarrow$

Note the analogy between eq. 5 and the definition of shortwave absorptivity (net shortwave radiative flux is given by the product of shortwave absorptivity and incoming shortwave flux density). It is not obvious that the reflection probability, as defined by eq. 5 , is correct when applied to reflection of individual trajectories, but that does seem to be the case.

To progress, we must evaluate or estimate $F_{r} \downarrow$, which must in general depend on many variables: time, location relative to the source and the details of the turbulence. Here, we will adopt a very simple model for $F_{r} \downarrow$. Decompose $w(t)$ and $c(t)$ into their respective mean and fluctuation values, $w(t)=\langle w\rangle+w^{\prime}$ and $c(t)=\langle c\rangle+c^{\prime}$. Define a correlation coefficient $\gamma(\gamma<0)$ by $\langle w c\rangle=\gamma \sigma_{w} \sigma_{c}$, where $\sigma_{c}$ is the standard deviation of the concentration fluctuation. We may model the time dependence of $w, c$ by

$w=w^{\prime}=\sigma_{w} r_{1}$

$c=\langle c\rangle+\gamma \sigma_{c} r_{1}+\left(1-\gamma^{2}\right)^{1 / 2} \sigma_{c} r_{2}$

where $r_{1}$ and $r_{2}$ are independent random numbers each drawn from a population having a Gaussian probability distribution with zero mean and unit variance. It is easily shown that the correct flux $\langle w c\rangle$ and variances result from this model. We may now evaluate the corresponding $F_{r} \downarrow$. Note that

$$
\left\langle w c \mid r_{1}\right\rangle=\sigma_{w}\langle c\rangle r_{1}+\gamma \sigma_{w} \sigma_{c} r_{1}^{2}
$$

so that

$$
\begin{aligned}
F_{r} \downarrow & =-\left\langle\left(w c \mid r_{1}<0\right)\right\rangle \\
& =-\int_{-\infty}^{0}\left[\sigma_{w}\langle c\rangle r_{1}+\gamma \sigma_{w} \sigma_{c} r_{1}^{2}\right] \frac{1}{(2 \pi)^{1 / 2}} c f 1 \exp \left[-r_{1}^{2} / 2\right] \mathrm{d} r_{1} \\
& =\sigma_{w}\langle c\rangle /(2 \pi)^{1 / 2}-0.5 \gamma \sigma_{w} \sigma_{c}
\end{aligned}
$$

Similarly

$F_{r} \uparrow=\sigma_{w}\langle c\rangle /(2 \pi)^{1 / 2}+0.5 \gamma \sigma_{w} \sigma_{c}$

It follows from eq. 9 a for $F_{r} \downarrow$ that the reflection probability is given by

$\frac{1-R}{1+R}=\left(\frac{\pi}{2}\right)^{1 / 2} \frac{w_{d}}{\sigma_{w}}$

The form of eq. 10 is most interesting. The magnitude of $w_{d}$ is known to be 
proportional to the friction velocity $u_{*}$ (and therefore $\sigma_{w}$ ) over a wide range of physical conditions and for many sink geometries (Chamberlain, 1966, 1967; Ferrandino and Aylor, 1985). Thus, the reflection probability may be relatively independent of the friction velocity and by implication relatively independent of atmospheric conditions. It will be. shown later that when eq. 10 for the reflection probability is implemented in numerical simulations, the correct deposition rate and concentration field are obtained.

\section{Discussion of the reflection rule and its implementation}

The above partial reflection rule is not rigorous. The assumed relationship between $w(t)$ and $c(t)$ is surely an over-simplification since only the first and second moments are correctly specified. The derivation yields a result which makes no reference to the nature of the stochastic process within which the reflection rule is to be imbedded and contains only a single variable, $\sigma_{w}$, pertaining to the nature of the turbulence. Thus, to the extent that the rule is valid, it should be useful for any type of stochastic model which is adopted, be it a crude model (such as a Markovian position process) of motion in homogeneous turbulence or a relatively detailed model (such as a Markovian model of the joint velocity-position process) of motion in the inhomogeneous turbulence adjacent to the absorbing boundary. The reflection rule is to be applied only to particles whose trajectories cross the reference plane $z_{r}$ (in contrast to an alternative rule given by Boughton et al. (1987) which will be discussed later) and is in principle applied at the instant of crossing (in a discrete time model, of course, this cannot be exactly adhered to). The derivation of eq. 10 as a reflection rule yields absolutely no information on how reflection should be implemented.

Let us now assume that the process within which the rule is to be imbedded is the joint particle position-velocity process $\left(z_{k}, w_{\mathrm{L} k}, t_{k}\right)$, which for time increments $\Delta t=t_{k}-t_{k-1}$ exceeding the particle acceleration time scale is Markovian. If the initial position is $z\left(t_{0}\right)=h$, then

$$
z\left(t_{i}\right)=h+\sum_{k=0}^{i-1} w_{\mathrm{L} k} \Delta t
$$

$t_{i}=i \Delta t$

where $w_{\mathrm{L} k}$, the Lagrangian velocity, is obtained from a suitable model, perhaps a Markov chain

$w_{\mathrm{L} k+1}=\alpha w_{\mathrm{L} k}+\beta \sigma_{w} r_{k+1}$

$w_{\mathrm{L} 1}=\sigma_{w} r_{1}$

Here, $r_{k}$ is drawn at random from a standard normal distribution, 
$\alpha=1-\Delta t / T_{\mathrm{L}}$ is the value of the velocity autocorrelation function for lag $\Delta t$, and $\beta=\left(1-\alpha^{2}\right)^{1 / 2}$.

The state of "encounter" at time $t_{k}$ (meaning that an encounter with the surface $z=z_{r}$ will occur between $t_{k}$ and $t_{k+1}$ ) is defined to be the event $\left[z_{k} \leqslant z_{r}-w_{\mathrm{L} k} \Delta t, w_{\mathrm{L} k}<0, t_{k}\right]$. An "escape" is the joint event

$\left[z_{k+1} \geqslant z_{r}, w_{\mathrm{L} k+1} \geqslant-\left(z_{k+1}-z_{r}\right) / \Delta t, t_{k+1} ; z_{k} \leqslant z_{r}-w_{\mathrm{L} k} \Delta t, w_{\mathbf{L} k}<0, \mathbf{t}_{k}\right]$

where the specified escape state must result from the intervention of a partial reflection rule consisting of a reflection probability and a reflection procedure; otherwise $z_{k+1}=z_{r}+w_{\mathrm{L} k} \Delta t \leqslant z_{r}$.

Two possible implementations of the escape state are

$$
\begin{aligned}
& \text { (i) } z_{k+1}=2 z_{r}-\left(z_{k}+w_{\mathrm{L} k} \Delta t\right) \\
& w_{\mathrm{L} k+1}=-\alpha w_{\mathrm{L} k}+\beta \sigma_{w} r_{k+1}
\end{aligned}
$$

Following Thurtell and Thurtell (1988), we call this "smooth wall reflection" (because the velocity upon reflection is correlated with the arrival velocity).

$$
\text { (ii) } z_{k+1}=z_{r} \quad w_{\mathrm{L} k+1}=\sigma_{w} r
$$

where the distribution of $r$ is

$$
\begin{aligned}
f_{r}(r) & =2 /(2 \pi)^{1 / 2} \exp \left[-r^{2} / 2\right] & & r \geqslant 0 \\
& =0 & & r<0
\end{aligned}
$$

("rough wall reflection").

On first thought, Rule (ii) might be preferred because we know that, in reality, a particle passing below $z_{r}$ usually has a "deep" layer (in the sense that many local time scales will elapse before arrival at the sink) beneath it and that if it does re-emerge its velocity will not be correlated with its entry velocity. However, the reflection procedure under discussion is a modelling convenience rather than an attempt to describe reality. The work of Thurtell and Thurtell (1988) suggests that Rule (ii) should not be used because in effect this means placing a source at $z=z_{r}$ so that "near-field" effects will result. For example, if Rule (ii) is implemented with $R=1$ (perfect reflection) the result will be a non-zero concentration gradient in a region having a depth of the order of 1 length scale near $z=z_{r}$ rather than, as desired, $(\partial\langle c\rangle / \partial z)_{z r}=0$.

\section{An alternative derivation based on the Fokker-Planck equation}

Let us consider the concentration field arising from an instantaneous area source of unit strength at height $z=h$, time $t=0$ in a steady-state, horizontally homogeneous field of turbulence, with partial absorption occurring at $z=z_{r}$. The Lagrangian description of the ensemble average concentration field resulting from an arbitrary source distribution $Q\left(x_{i}, t_{i}\right)$ is 
$C(x, t)=\int_{\text {all }} \int_{x_{i}}^{t} Q\left(\underset{t_{i}}{x_{i}}, t_{i}\right) p\left(\underset{\sim}{x}, t \mid x_{i}, t_{i}\right) \mathrm{d}{\underset{\sim}{x}}_{i} \mathrm{~d} t_{i}$

where $\mathrm{d} x_{i}=\mathrm{d} x_{i} \mathrm{~d} y_{i} \mathrm{~d} z_{i}$. To use this expression, we need to know the probability density function (pdf) $p\left(x, t \mid x_{i}, t_{i}\right)$ for fluid element position (conditioned on release time and position). Lagrangian stochastic methods are commonly used to evaluate this pdf, and we will adopt eq. 11 for this purpose. For the specified source distribution $Q\left(x_{i}, t_{i}\right)=\delta\left(z_{i}, h\right) \quad \delta\left(t_{i}, 0\right)$, eq. 14 reduces to $C(z, t)=p(z, t \mid h, 0)$.

Corresponding to the choice of eq. 11 to calculate trajectories is the FokkerPlanck equation (Van Dop et al., 1985)

$\frac{\partial}{\partial t} p\left(z, w_{\mathrm{L}}, t \mid h, 0\right)=-\frac{\partial J_{z}}{\partial z}-\frac{\partial J_{w}}{\partial w_{\mathrm{L}}}$

which gives the time evolution of the joint position-velocity pdf $p\left(z, w_{\mathrm{L}}, t \mid h, 0\right)$. $J_{z}, J_{w}$ are, respectively, the fluxes of probability density along the $z, w$ axes of state space and are given by

$J_{z}\left(z, w_{\mathrm{L}}, t \mid h, 0\right)=w_{\mathrm{L}} p\left(z, w_{\mathrm{L}}, t \mid h, 0\right)$

$J_{w}\left(z, w_{\mathrm{L}}, t \mid h, 0\right)=-\frac{w_{\mathrm{L}} p\left(z, w_{\mathrm{L}}, t \mid h, 0\right)}{T_{\mathrm{L}}}-\frac{\sigma_{w}{ }^{2}}{T_{\mathrm{L}}} \frac{\partial p\left(z, w_{\mathrm{L}}, t \mid h, 0\right)}{\partial w_{\mathrm{L}}}$

An initial condition and boundary conditions at $z=0, z_{r}$ and $w_{\mathrm{L}}= \pm \infty$ must be given to completely specify the problem.

It may be shown that the ensemble average flux density at $z, t$ is

$$
\begin{aligned}
\langle w c\rangle & =\int_{-\infty}^{\infty} w_{\mathrm{L}} p\left(z, w_{\mathrm{L}}, t \mid h, 0\right) \mathrm{d} w_{\mathrm{L}} \\
& =\int_{-\infty}^{\infty} J_{z}\left(z, w_{\mathrm{L}}, t \mid h, 0\right) \mathrm{d} w_{\mathrm{L}} \\
& =C(z, t)\left\langle w_{\mathrm{L}}(z, t \mid h, 0)\right\rangle
\end{aligned}
$$

where an ensemble average Lagrangian velocity for particles which are at $z$ at time $t$, having been released at $h$ at $t=0$ has been defined by

$$
\begin{aligned}
\left\langle w_{\mathrm{L}}(z, t \mid h, 0)\right\rangle & =\int_{-\infty}^{\infty} J_{z}\left(z, w_{\mathrm{L}}, t \mid h, 0\right) \mathrm{d} w_{\mathrm{L}} / \int_{-\infty}^{\infty} p\left(z, w_{\mathrm{L}}, t \mid h, 0\right) \mathrm{d} w_{\mathrm{L}} \\
& =\int_{-\infty}^{\infty} w_{\mathrm{L}} p\left(z, w_{\mathrm{L}}, t \mid h, 0\right) \mathrm{d} w_{\mathrm{L}} / C(z, t)
\end{aligned}
$$


What are the boundary conditions to be applied to eq. 15 at $z=z_{r}$ ? The correct depositional flux is ensured by imposing

$$
\begin{aligned}
\int_{-\infty}^{\infty} J_{z}\left(z_{r}, w_{\mathrm{L}}, t \mid h, 0\right) \mathrm{d} w_{\mathrm{L}} & =\left\langle w_{\mathrm{L}}\left(z_{r}, t \mid h, 0\right)\right\rangle C_{r} \\
& =-w_{d} C_{r}
\end{aligned}
$$

i.e. we must ensure

$$
\left\langle w_{\mathrm{L}}\left(z_{r}, t \mid h, 0\right)\right\rangle=-w_{d}
$$

As one would expect, the ensemble mean particle velocity at $z=0$ is negative whereas the mean Eulerian velocity is zero.

Now it follows from the definition of $J_{w}$ and eq. $19 \mathrm{~b}$ that

$$
\int_{-\infty}^{\infty} J_{w}\left(z_{r}, w_{\mathrm{L}}, t \mid h, 0\right) \mathrm{d} w_{\mathrm{L}}=-w_{d} C_{r} / T_{\mathrm{L}}
$$

This states that if $w_{d}=0$ (a perfectly reflecting boundary at $z=z_{r}$ ), the efflux of probability density with $w_{\mathrm{L}}<0$ must be exactly balanced by an influx of probability density with $w_{\mathrm{L}}>0$, and the centre of mass must rise with increasing $t$. This may be achieved, for example, by reflection according to eq. $12 \mathrm{a}$ and b; a particle arriving at $z=0$ with $w_{\mathrm{L}}=w_{\mathrm{L} 1}<0$ must leave with velocity $w_{\mathrm{L}}=-w_{\mathrm{L} 1}$.

The general solution to the Fokker-Planck eq. 15 may be difficult to obtain and, in any case, we have not yet specified the exact boundary conditions on $p\left(z, w_{\mathrm{L}}, t \mid h, 0\right)$ at $z=z_{r}$ (which presumably depend on the precise reflection strategy adopted). Since we have only the integral condition eq. 19a, we will integrate the Fokker-Planck equation with respect to $w_{\mathrm{L}}$ to obtain

$$
\frac{\partial}{\partial t} p(z, t \mid h, 0)=-\frac{\partial}{\partial z}\left[\left\langle w_{\mathrm{L}}(z, t \mid h, 0)\right\rangle p(z, t \mid h, 0)\right]
$$

By further integrating with respect to $z$, we obtain

$$
\begin{aligned}
\frac{\partial}{\partial t} \int_{z_{r}}^{\infty} p(z, t \mid h, 0) \mathrm{d} z & =\left[\left\langle w_{\mathrm{L}}(z, t \mid h, 0)\right\rangle p(z, t \mid h, 0)\right]_{\infty}^{z_{r}} \\
& =\left\langle w_{\mathrm{L}}\left(z_{r}, t \mid h, 0\right)\right\rangle C_{r}
\end{aligned}
$$

which confirms the requirement on the mean Lagrangian velocity expressed by eq. $19 \mathrm{~b}$; it may be that this requirement is intuitively obvious.

We may progress further by specifying that smooth wall reflection will be implemented. Then the reflection probability is defined by 
$p\left(z_{r},-w_{\mathrm{L}}, t \mid h, 0\right)=R p\left(z_{r}, w_{\mathrm{L}}, t \mid h, 0\right) \quad$ for all $\quad w_{\mathrm{L}}<0$

In words, this prescription states that "a particle at $z=z_{r}$ having $w_{\mathrm{L}}<0$ will with probability $R$ survive at $z=z_{r}$ with its velocity reversed, and any particle which is at $z=z_{r}$ with $w_{\mathrm{L}}>0$ is in a "survival" state immediately following the state of encounter".

We may now evaluate $\left\langle w_{\mathbf{L}}\left(z_{r}, t \mid h, 0\right)\right\rangle=-w_{d}$ by using eq. 23 in eq. 18 . Splitting any integral of the form $\int_{-\infty}^{\infty}() \mathrm{d} w_{\mathrm{L}}$ into $\int_{-\infty}^{0}() \mathrm{d} w_{\mathrm{L}}+\int_{0}^{\infty}() \mathrm{d} w_{\mathrm{L}}$ we obtain

$\frac{1-R}{1+R}=-\frac{w_{d}}{\left\langle w_{\mathrm{L}} \downarrow\right\rangle}$

where $\left\langle w_{\mathrm{L}} \downarrow\right\rangle$ is the average velocity of particles in the encounter state

$$
\left\langle w_{\mathrm{L}} \downarrow\right\rangle=\int_{-\infty}^{0} w_{\mathrm{L}} p\left(z_{r}, w_{\mathrm{L}}, t \mid h, 0\right) \mathrm{d} w_{\mathrm{L}} / \int_{-\infty}^{0} p\left(z_{r}, w_{\mathrm{L}}, t \mid h, 0\right) \mathrm{d} w_{\mathrm{L}}
$$

It is easy to show that the average velocity of the (less numerous) particles in the survival state is $\left\langle w_{\mathrm{L}} \uparrow\right\rangle=-\left\langle w_{\mathrm{L}} \downarrow\right\rangle$ and this conforms with intuition.

We have recovered a reflection rule which is identical in form to eq. 10 , but the pdf for the vertical velocity in the encounter state is unknown. If it is assumed that the encounter velocity has a one-sided normal distribution

$$
\begin{aligned}
f\left(w_{\mathrm{L}} \downarrow\right) & =\frac{2}{(2 \pi)^{1 / 2} \sigma_{w}} \exp \left[-w_{\mathrm{L}} \downarrow^{2} / 2 \sigma_{w}{ }^{2}\right] & & w_{\mathrm{L}} \downarrow<0 \\
& =0 & & w_{\mathrm{L}} \downarrow>0
\end{aligned}
$$

we recover eq. 10 .

Aylor and Ferrandino (1989) obtained an equation very similar to eq. $10 \mathrm{~b}$. Their equation (Alla), modified for the case of diffusive rather than sedimentary uptake, implies a variable reflection probability $R=R\left(w_{d}, w_{\mathbf{L}} \downarrow\right)$ which depends on the (variable) encounter velocity $w_{\mathrm{L}} \downarrow$

$\frac{1-R}{1+R}=-\frac{w_{d}}{w_{\mathrm{L}} \downarrow}$

Before progressing, it is of interest to define conditional mean concentrations at the reflection height

$$
C_{r} \uparrow=\int_{0}^{\infty} p\left(z_{r}, w_{\mathrm{L}}, t \mid h, 0\right) \mathrm{d} w_{\mathrm{L}}
$$


$C_{r} \downarrow=\int_{-\infty}^{0} p\left(z_{r}, w_{\mathbf{L}}, t \mid h, 0\right) \mathrm{d} w_{\mathrm{L}}$

It follows from eq. 25 that $C_{r} \uparrow=R /(1+R) C_{r}$ and $C_{r} \downarrow=1 /(1+R) C_{r}$. At a perfectly absorbing surface $(R=0)$, there are no particles moving away from the surface, but the mean concentration is non-zero, as it must be if there is to be a flux to the surface. This conflicts with the common practise of applying to the Fickian diffusion equation a boundary condition of zero concentration at a perfectly absorbing wall.

\section{ALTERNATIVE APPROACHES}

Boughton et al. (1987; hereafter BDD) have modelled dispersion in homogeneous and inhomogeneous turbulence above a partially absorbing surface as a Markovian stochastic process in position. The absorbing surface was treated as an extended sink. Here, we will discuss their treatment of absorption for a non-buoyant substance in homogeneous turbulence with the nominal sink at $z=0$.

Let $p\left(z_{2}, t_{2} \mid z_{1}, t_{1}\right)$ denote the particle position probability density at time $t_{2}$ given that at $t_{1}$ the particle is at $z_{1}$. If at time $t_{i}$ the particle is at $z_{i}$, close to the (absorbing) surface at $z=0$, then

$1-A=\int_{0}^{\infty} p\left(z, t_{i}+\Delta t \mid z_{i}, t_{i}\right) \mathrm{d} z$

is the probability that the particle has "survived" the succeeding interval $\Delta t$ and is still therefore to be found above $z=0$. This integral may be evaluated as the solution to the equation

$\frac{\partial p}{\partial t}=K \frac{\partial^{2} p}{\partial z^{2}}$

subject to the lower boundary condition

$$
\left(K \frac{\partial p}{\partial z}-w_{d} p\right)_{z=0}=0
$$

and the initial condition of a unit release at $z=z_{i}, t=t_{i}$. The solution is

$A=\operatorname{erfc}\left[\frac{z_{i}}{2(K \Delta t)^{1 / 2}}\right]-\exp \left[\frac{z_{i} w_{d}+w_{d}^{2} \Delta t}{K}\right] \operatorname{erfc}\left[\frac{z_{i}+2 w_{d} \Delta t}{2(K \Delta t)^{1 / 2}}\right]$

Note that the BDD rule does contain a variable which depends on the nature 
of the stochastic process adopted as a model, namely the time step $\Delta t$. The $\mathrm{BDD}$ reflection procedure is:

(i) Add random position increment $\Delta z$ to the current position $z_{i}$ to obtain $z_{i}+\Delta z$.

(ii) Evaluate eq. 29 for the given $\Delta t, z_{i}$ to find the likelihood, $A$, that the particle would have been absorbed during the interval $\Delta t$ according to the analytical solution.

(iii) If a number chosen randomly from the uniform distribution [0,1] does not exceed $A$, the particle is absorbed. Otherwise, the particle is kept and if $z_{i}+\Delta z<0$ the particle is reflected to $-\left(z_{i}+\Delta z\right)$.

There is a non-zero (though very small for large $z_{i}$ ) probability of absorption even if $z_{i}+\Delta z>0$, i.e., even if the calculated step in position does not take the particle across the absorption plane at $z=0$. Therefore, in principle, the BDD absorption decision must be made at every time step and the absorbing plane is in effect treated as an extended sink. The "reflection probability" $R=1-A$ therefore has a different meaning from that given by eq. 10 and is in reality a survival probability.

Another extended sink treatment which has been found to yield correct depositional flux and reference concentration is to absorb the particle if, at the end of the time step, it lies within the layer $z_{r} \pm w_{d} \Delta t$ and reflect if it lies below $z_{r}-w_{d} \Delta t$. No justification will be given for this method, which has not been exhaustively tested. The necessary conditions are $w_{d} \ll \sigma_{w}$ and $\Delta t \ll T_{\mathrm{L}}$.

\section{NUMERICAL VERIFICATION OF THE REFLECTION RULE}

The new partial reflection rule eq. 10 has been implemented in simulations in order to verify that it yields the correct relationship between depositional flux and concentration at the reference height. A particle crossing the reference height was reflected if the ratio $n_{r} / n_{0}$ of all earlier reflections to all surface encounters did not exceed the desired ratio $R$. Results will be given for two diffusion problems.

\section{Elevated area source in the neutral surface layer}

The source was placed at dimensionless height $h / z_{0}=100$ (where $z_{0}$ is the surface roughness length) and extended upstream from the "measurement point" a distance $x / z_{0}=50000$. The partially absorbing surface was placed at $z / z_{0}=1$. Trajectories were simulated essentially according to eqs. 11 and 12 , except that the " $z_{*}-t_{\mathrm{H}}$ " coordinate transformation described by Wilson et al. (1981) was employed. The Lagrangian time scale was specified as $T_{\mathrm{L}}=0.5 \mathrm{z} /$ $\sigma_{w}$ and the time step as $0.2 T_{\mathrm{L}}$. Depositional flux was averaged over a length $\mathrm{d} x / z_{0}=100$ at the measurement location, and the streamwise flux (whence mean concentration is derived) was averaged over height intervals $\mathrm{d} z / z_{0}$ which 
were small in comparison with the local length scale. The streamwise velocity was calculated from the logarithmic mean wind profile.

As the reflection probability $R$ becomes small, it becomes difficult to estimate the surface concentration, $C_{r}$, accurately due to the strong vertical concentration gradient near the surface. When necessary (in practise for $R \leqslant 0.5$ ), to avoid having this problem compromise the test of the reflection rule, the effective deposition velocity has been derived from the concentration at $z$ / $z_{0}=10$ by subtracting from the effective deposition resistance $r_{\text {tot }}$ based on $\left\langle c\left(z / z_{0}\right)=10\right\rangle$, the aerodynamic resistance $r_{\mathrm{a}}$ between $z_{0}$ and $z=10 z_{0}$, i.e.

$\frac{1}{w_{d}}=r_{\mathrm{tot}}-r_{\mathrm{a}}=\frac{\langle c(10)\rangle}{F_{r}}-r_{\mathrm{a}}$

where

$r_{\mathrm{a}}=\int_{z_{0}}^{10 z_{0}} \frac{\mathrm{d} z}{0.5 \sigma_{w} z}=\frac{\ln 10}{0.5 \sigma_{w}}$

Table 1 shows the mean value and standard error of the (normalised) effective deposition velocity $w_{d} / \sigma_{w}$ obtained from the simulations as a function of the nominal value $w_{d} / \sigma_{w}$ used to calculate the reflection probability $R$ (using eq. 10). The standard error was evaluated from eight repetitions each of 3000 releases. Over a range of three orders of magnitude in $w_{d} / \sigma_{w}$, there is excellent correspondence between the actual and desired deposition velocities. The small discrepancy for $R<0.5$ may be due to the fact that eq. 31 underestimates the aerodynamic resistance because it does not account for near-field effects. Because such large values of $w_{d} / \sigma_{w}$ are not encountered in reality, we have not pursued this minor point.

\section{TABLE 1}

Outcome of simulations of dispersion in the neutral atmospheric surface layer with partial absorption at ground. $R$ is the reflection probability and $\left(w_{d} / \sigma_{w}\right)^{\text {theoretical }}$ is the corresponding normalised deposition velocity, calculated using eq. 10. $\left(w_{d} / \sigma_{w}\right)^{\text {simulation }}$ is the effective normalised deposition velocity following from the depositional flux and concentration calculated by the numerical simulation

\begin{tabular}{lll}
\hline$R$ & ${\frac{w_{d}}{\sigma_{u}}}^{\text {theuretical }}$ & ${\frac{w_{d}}{\sigma_{u}}}^{\text {simulation }} \pm$ std. error \\
\hline 0.99971 & $1.16 \times 10^{-4}$ & $(1.12 \pm 0.15) \times 10^{-4}$ \\
0.995 & $2.00 \times 10^{-3}$ & $(1.99 \pm 0.03) \times 10^{-3}$ \\
0.95 & $2.05 \times 10^{-2}$ & $(2.06 \pm 0.03) \times 10^{-2}$ \\
0.50 & $2.66 \times 10^{-1}$ & $(2.41 \pm 0.01) \times 10^{-1}$ \\
0.20 & $5.32 \times 10^{-1}$ & $(4.93 \pm 0.04) \times 10^{-1}$ \\
\hline
\end{tabular}




\section{Vertical dispersion in homogeneous turbulence}

Carslaw and Jaeger (1959; p. 358) give the following analytical solution for the ensemble-mean time-dependent concentration $\langle c(z, t)\rangle$ resulting from the release at height $z=h$ of a unit sheet of a neutrally buoyant species into homogeneous turbulence characterised by eddy diffusivity $K$, with a partially absorbing boundary at $z=0$ causing a deposition velocity $w_{d}$

$$
\begin{aligned}
\langle c(z, t)\rangle & =\frac{1}{2(\pi K t)^{1 / 2}}\left\{\exp \left[-\frac{(z+h)^{2}}{4 K t}\right]+\exp \left[-\frac{(z-h)^{2}}{4 K t}\right]\right\} \\
& -\frac{w_{d}}{K} \exp \left[\frac{w_{d}(z+h)+w_{d}^{2} t}{K}\right] \operatorname{erfc}\left[\frac{z+h+2 w_{d} t}{2(K t)^{1 / 2}}\right]
\end{aligned}
$$

In reality, the presence of a sink would imply a solid boundary at $z=0$ and therefore homogeneous turbulence bounded by a sink is not realisable. Furthermore, eq. 32 is incorrect for small $t$ due to near-field effects. However, simulations using a stochastic joint position-velocity process (with appropriate position-independent velocity statistics) should agree with eq. 32 provided $t$ is not too small (a stochastic process in position alone should agree at all times provided reflection is treated in a manner consistent with the analytical treatment).

Figure 2 shows the result of a simulation of this system using exactly the position-velocity process specified by eqs. 11 and 12 . The turbulence was specified as $\sigma_{w}=1, T_{\mathrm{L}}=1(K=1)$ and the "particles" (actually sheets) were released at $h=10$. Ensemble mean concentration was estimated in height-time windows of depth $\Delta z=1$ and short duration (relative to time since release) for simulations with $R=(0.95,0.5,0.2)$. The analytical solutions were obtained by integration of eq. 32

$$
\begin{aligned}
\int_{0}^{z t}\langle c(z, t)\rangle \mathrm{d} z & =\frac{1}{2}\left[\operatorname{erf}\left(\frac{z t-h}{2(K t)^{1 / 2}}\right)-\operatorname{erf}\left(\frac{z t+h}{2(K t)^{1 / 2}}\right)\right]+\operatorname{erf}\left(\frac{h}{2(K t)^{1 / 2}}\right) \\
& +\exp \left[\frac{w_{d} h+w_{d}^{2} t}{K}\right] \operatorname{erf}\left[\frac{h+2 w_{d} t}{2(K t)^{1 / 2}}\right] \\
& -\exp \left[\frac{w_{d}(z t+h)+w_{d}^{2} t}{K}\right] \operatorname{erfc}\left[\frac{z t+h+2 w_{d} t}{2(K t)^{1 / 2}}\right]
\end{aligned}
$$

with $z t=1$. The deposition velocity, $w_{d}$, corresponding to the reflection probability, $R$, used in each simulation follows from the reflection rule (eq. 10).

As expected, the joint position-velocity process gives a (presumably correct) solution differing from the diffusion equation solution at small $t$. The excellent agreement at large $t$ for all $R$ suggests that the reflection rule (eq. 10) is useful. 


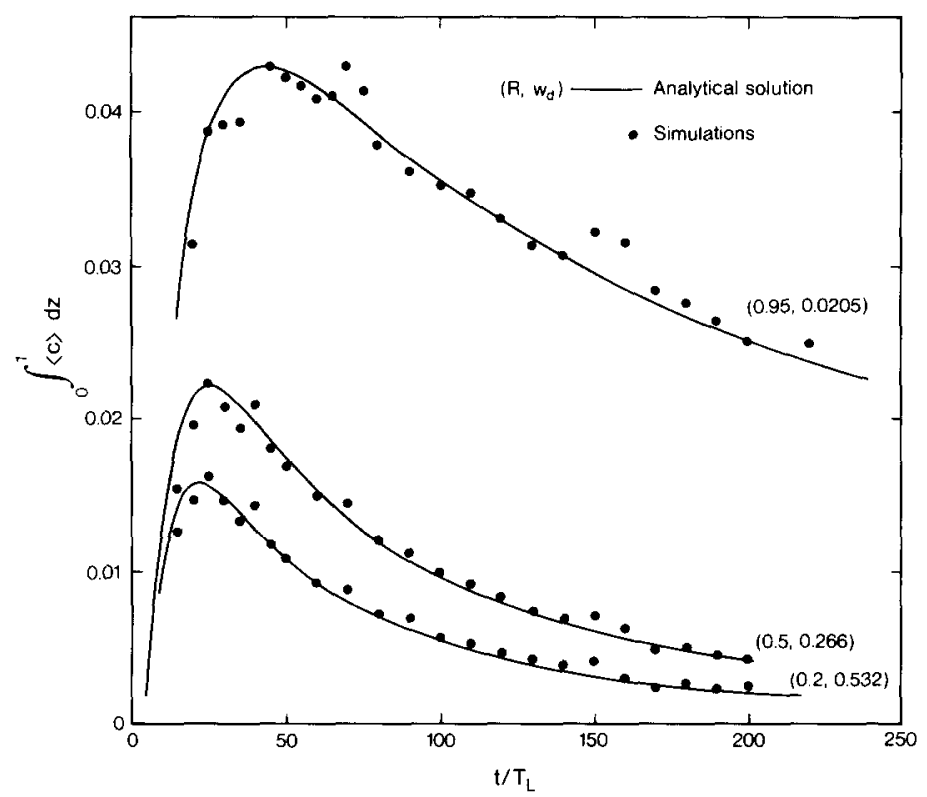

Fig. 2. Ensemble mean concentration versus time for an instantaneous elevated area source in homogeneous turbulence bounded at $z=0$ by a partially absorbing surface. The velocity, time and length scales of the turbulence are all unity and the source lies at $z=h=10$. The analytical solution (eq. 33) for the concentration integral $\int_{0}^{1}\langle c(z, t)\rangle \mathrm{d} z$ is plotted against estimates derived by numerical simulation of the process.

\section{CONCLUSION}

The simple reflection rule (eq. 10) has been shown to give the correct depositional flux and reference concentration over a wide range of values of the reflection probability (i.e., over a wide range in deposition velocity). Though the derivation of the rule is not rigorous, the successful outcome of simulations suggests that it cannot differ greatly from the "correct" rule. The earlier rule, eq. 10c due to Aylor and Ferrandino (1989), is probably equally satisfactory. We have given no proof that the appropriate implementation of reflection is smooth wall reflection (velocity reversal upon reflection), but we believe this to be the case.

\section{REFERENCES}

Aylor, D.E. and Ferrandino, F.J., 1989. Dispersion of spores released from an elevated line source within a wheat canopy. Boundary-Layer Meteorol., 46: 251-273. 
Boughton, B.A., Delaurentis, J.M. and Dunn, W.E., 1987. A stochastic model of particle dispersion in the atmosphere. Boundary-Layer Meteorol., 40: 147-163.

Carslaw, H.S. and Jaeger, J.C., 1959. Conduction of heat in solids, 2nd edn., Oxford University Press, Oxford.

Chamberlain, A.C., 1966. Transport of gases to and from grass and grass-like surfaces. Proc. R. Soc., 290A: 236-265.

Chamberlain, A.C., 1967. Transport of Lycopodium spores and other small particles to rough surfaces. Proc. R. Soc., 296A: 45-70.

Ferrandino, F.J. and Aylor, D.E., 1985. An explicit equation for the deposition velocity. BoundaryLayer Meteorol., 31: 197-201.

Fisher, B.E.A., 1983. A review of the processes and models of long-range transport of air pollutants. Atmos. Environ., 17: 1865-1880.

Sawford, B.L., 1986. Generalized random forcing in random-walk turbulent dispersion models. Phys. Fluids, 29: 3582-3585.

Sawford, B.L. and Guest, F.M., 1987. Lagrangian stochastic analysis of flux-gradient relationships in the convective boundary layer. J. Atmos. Sci., 44: 1152-1165.

Smith, F.B., 1983. Long-range transport of air pollution. Meteorol. Mag., 112: 237-244.

Thomson, D.J., 1987. Criteria for the selection of stochastic models of particle trajectories in turbulent flows. J. Fluid Mech., 180: 529-556.

Thurtell, G.W. and Thurtell, J., 1988. Adsorption and diffusion at rough surfaces: a comparison of statistical mechanics, molecular dynamics, and kinetic theory. J. Chem. Phys., 88: 66416646.

Van Dop, H., Nieuwstadt, F.T.M. and Hunt, J.C.R., 1985. Random walk models for particle displacements in inhomogeneous unsteady turbulent flows. Phys. Fluids, 28: 1639-1653.

Wilson, J.D., Thurtell, G.W. and Kidd, G.E., 1981. Numerical simulation of particle trajectories in inhomogeneous turbulence. I. Systems with constant turbulent velocity scale. BoundaryLayer Meteorol., 21: 295-313. 